\title{
Planar THz quasioptics
}

\author{
Jianming Dai, S. Coleman, and D. Grischkowsky ${ }^{a}$ ) \\ School of Electrical and Computer Engineering, Oklahoma State University, Stillwater, Oklahoma 74078
}

(Received 16 March 2004; accepted 11 June 2004)

\begin{abstract}
Two-dimensional (2D) quasioptical elements are demonstrated as guiding structures in the $\mathrm{THz}$ frequency regime. A planar polyethylene lens is incorporated in a parallel metal plate structure and is shown to focus guided $\mathrm{THz}$ radiation to a spot comparable to that expected for a three-dimensional (3D) optical element in free space. A 2D double-slit aperture is also demonstrated to diffract the incoming $\mathrm{THz}$ beam as expected for 3D. The spatial resolution of the measurement techniques employed is illustrated by the sharpness of the measured diffraction pattern. (C) 2004 American Institute of Physics. [DOI: 10.1063/1.1781357]
\end{abstract}

Parallel-plate metal waveguides have been demonstrated to exhibit low-loss transverse-electromagnetic (TEM)-mode propagation for frequencies up to $4.5 \mathrm{THz}$ and lengths to $0.25 \mathrm{~m} .{ }^{1,2}$ The utility of these devices has recently been extended by incorporating planar quasioptical flat mirrors between the plates yielding a low-loss TEM-mode guiding structure capable of guiding a $\mathrm{THz}$ pulse in both directions transverse to the direction of propagation. ${ }^{3}$ Furthermore, a method to optoelectronically excite guided modes in these structures has recently been developed. ${ }^{4}$ In this work, we extend the previous investigations by incorporating transmissive and diffractive quasioptical elements within the parallel plate structure. We demonstrate a planar lens for focusing the TEM-mode guided $\mathrm{THz}$ to a small spot within the guiding structure. This demonstration is related to earlier work at microwave frequencies, where planar quasioptical elements were incorporated within a slab waveguide. ${ }^{5}$ We also show that a double slit aperture placed at the input of this lens places the far-field diffraction pattern of the aperture at the lens focal plane. Thus, two-dimensional (2D) reflective, transmissive, and diffractive quasioptical elements are shown to work as their three-dimensional (3D) counterparts in freespace for the TEM mode of the guiding structure. The 2D quasioptics combined with the parallel plate guiding structure act as a 2D optical table, potentially allowing for efficient point-to-point $\mathrm{THz}$ pulse communication.

The experimental setup of Fig. 1 utilizes the standard confocal $\mathrm{THz}$ time-domain spectroscopy (THz-TDS) arrangement described elsewhere. ${ }^{6}$ The $2 \mathrm{D}$ quasioptical structure is placed at the confocal $\mathrm{THz}$ beam waist midway between the off-axis paraboloidal mirrors. This structure consists of a cylindrical silicon lens, a 2D low-density polyethylene (LDPE) lens incorporated within a parallel metal plate structure, and a silicon spherical output-coupling lens. The input cylindrical lens is $15 \mathrm{~mm} \times 10 \mathrm{~mm} \times 6.56 \mathrm{~mm}$ with a $5 \mathrm{~mm}$ radius of curvature and serves to couple the freely propagating beam of $\mathrm{THz}$ radiation to the transverse electromagnetic (TEM) mode of the interconnect structure. This lens focuses the incoming $\mathrm{THz}$ to an elliptical spot with a frequency independent minor axis with $1 / e$ amplitude diameter of $200 \mu \mathrm{m}$ in the $y$ direction and a linearly wavelength-dependent major axis with a $1 / e$ amplitude diameter of $9 \mathrm{~mm}$ in the $x$ direction at $1 \mathrm{THz}$. The minor axis

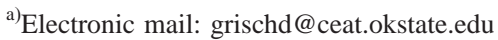

and the field polarization are perpendicular to the 2D layer between the plates. These plates are fabricated from polished oxygen free copper alloy 101. The individual plate dimensions are $56 \mathrm{~mm} \times 56 \mathrm{~mm} \times 9.5 \mathrm{~mm}$. The plates are separated by the $146 \mu \mathrm{m}$ thickness of the LDPE lens. The lens is $41 \mathrm{~mm} \times 10 \mathrm{~mm}$ with a $26.4 \mathrm{~mm}$ radius of curvature. The focal length of the lens can be calculated as for a standard thick lens, with the radius of one side set to infinity: $f$ $=R /(n-1)$, where $R$ is the radius of curvature of the lens $(26.4 \mathrm{~mm})$, and $n=1.51$ is the index of the lens material. ${ }^{7}$ These values yield a focal length of $f=51.8 \mathrm{~mm}$ for the described LDPE lens. However, this focal length is measured from the second principal plane of the lens, $3.4 \mathrm{~mm}$ into the lens from the vertex of the curved input face which is aligned to the input edge $(z=0)$ of the 2D structure. Consequently, the paraxial focus of our lens lies within $1 \mathrm{~mm}$ of the output edge $(z=56 \mathrm{~mm})$ of the test structure.

The output edge is also at the focus of a hyperhemispherical Si lens with $5 \mathrm{~mm}$ radius and $6.56 \mathrm{~mm}$ lens height. This output lens point samples the field as a function of $x$ position along the output edge, thereby coupling a localized portion of the guided mode into the receiver. In order

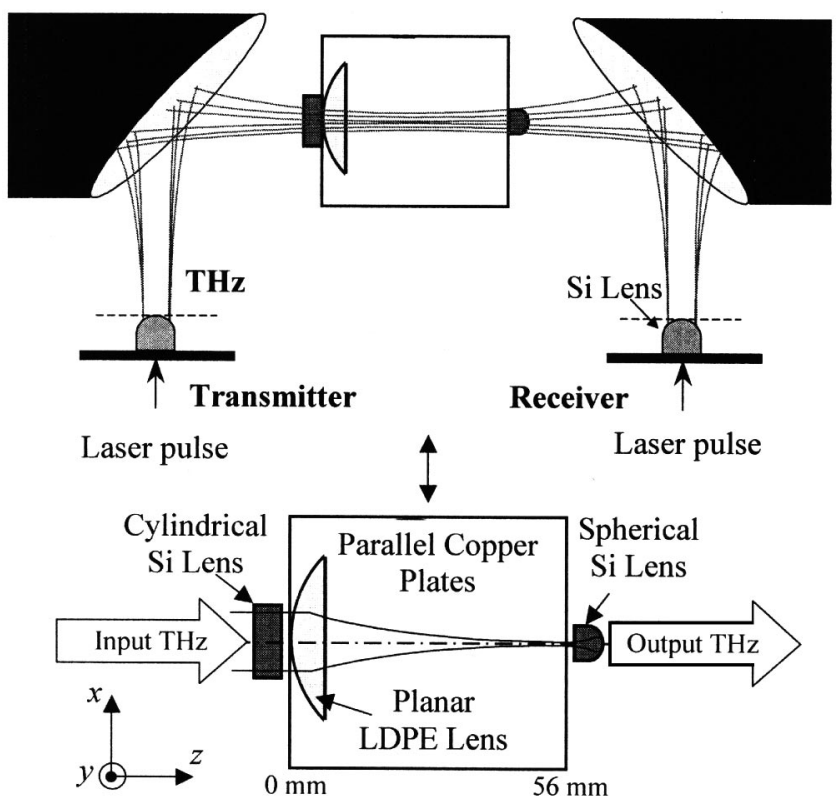

FIG. 1. THz-TDs arrangement and 2D quasioptical test structure. 

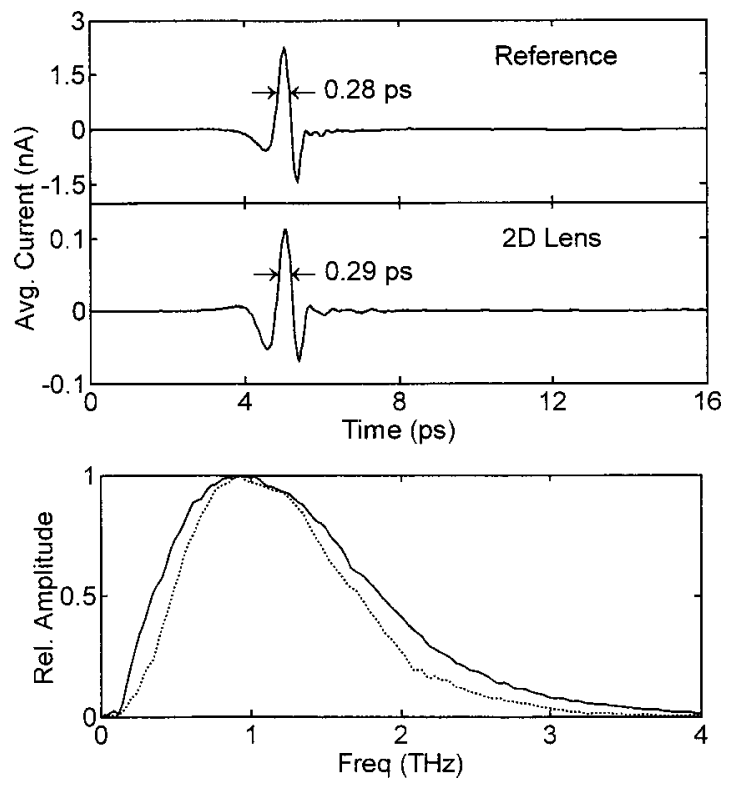

FIG. 2. Reference and signal THz pulses, arrows indicate FWHM pulse width. Relative amplitude spectra for the reference pulse (solid line) and signal pulse (dashed line). Signal pulse spectrum multiplied by 16.5 to facilitate comparison.

to obtain this spatial measurement of the $\mathrm{THz}$ electric field, the cylindrical input and spherical output lenses are held stationary, as shown in Fig. 1, while the remainder of the test structure is translated along the $x$ axis. For each incremental translation of the test structure, a measurement is made of the $\mathrm{THz}$ pulse coupled through the device.

For this experiment, the 2D test structure and all associated lenses were first removed. The $\mathrm{THz}$ signal was optimized, and a reference pulse was taken with the system in the standard confocal configuration. ${ }^{6}$ The test structure was then inserted into the $\mathrm{THz}$ beam path and the measured signal was again maximized. A second scan was then taken of the $\mathrm{THz}$ signal pulse corresponding to the center $(x=0)$ of the focal spot produced by the LDPE lens. The reference $\mathrm{THz}$ pulse and the $(x=0)$ signal pulse are shown in the upper panel of Fig. 2. The fact that the two full-width at halfmaximum (FWHM) measured pulse widths vary by less than 10 fs illustrates the minimal phase and group velocity dispersion of the entire 2D structure. This performance is due to the TEM-mode propagation through the test structure and the precision fabrication of the LDPE lens. The lower panel of this figure compares the amplitude spectrum of each pulse, where the spectrum of the signal pulse is multiplied by the factor 16.5 for comparison. The two spectra are quite similar except for the signal spectrum showing more attenuation at the frequency extremes. The smoothness of the signal spectrum with no observed low-frequency cutoffs confirms that the detected signal pulse propagates in the TEM mode of the structure. With the test structure in place, the focusing action of the 2D LDPE lens is determined by taking a THz signal pulse measurement for each incremental movement of the structure along the $x$ axis. The measured data are shown for three different frequencies in Fig. 3, obtained from the amplitude spectra of the corresponding signal pulses.

In order to understand our measurements, we use standard Gaussian beam propagation theory to calculate that at the input face of the cylindrical lens the 1/e input amplitude waist diameters are $D_{e}=18.4,9.2$, and $4.6 \mathrm{~mm}$ at $0.5,1$, and Downloaded 23 Sep 2004 to 129.110.241.62. Redistribution subject

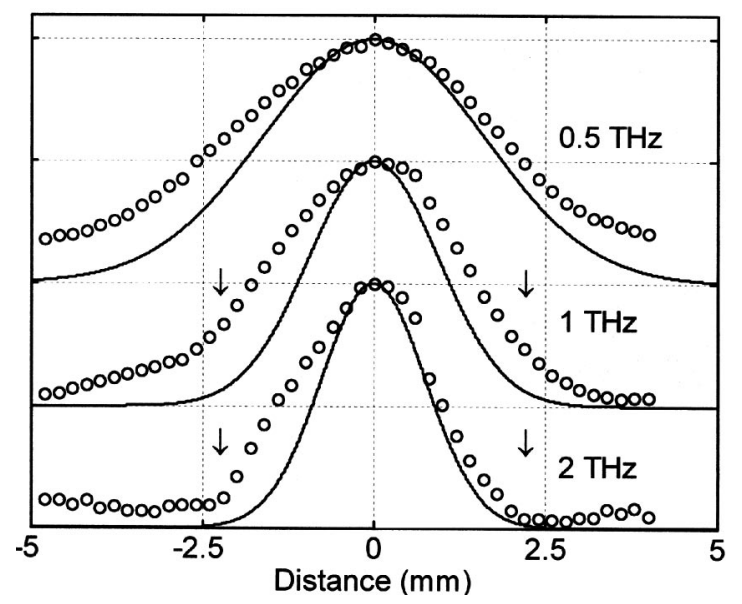

FIG. 3. Measured spatial cross section of the focus at the output edge of the $\mathrm{THz}$ electric field (normalized amplitudes) at various frequencies (open circles) and the calculated field dependence (solid lines).

$2 \mathrm{THz}$, respectively. Independent measurements on a similar THz-TDS system of the signal transmission through an adjustable aperture located at the confocal position yields $1 / e$ amplitude diameters of $D_{e}=15,8.5$, and $4.25 \mathrm{~mm}$ at $0.5,1$, and $2 \mathrm{THz}$, respectively. These measurements also indicated that the beam profiles had sharper edges and more complex shapes than a simple Gaussian, in agreement with recent work. ${ }^{8}$ If for the moment we neglect these beam complications, aperturing effects, and spherical aberration from the 2D lens, the incoming $\mathrm{THz}$ radiation should be focused to a frequency independent spot at the output edge of the test structure, according to the Gaussian beam relationship $d_{e}$ $=4 f \lambda /\left(\pi D_{e}\right)$, where $d_{e}$ is the focused $1 / e$ beam waist diameter. For our case this diameter should be $2.15 \mathrm{~mm}$. However, the measured beam waists, depicted in Fig. 3 by open circles, are clearly frequency dependent, indicating that the above effects may be significantly affecting the measured signals.

The input cylindrical lens presents a 12-mm-diameter clear aperture to the input field patterns. Thus, the actual field pattern at the input of the lens is the product of the lens clear aperture and the unapertured frequency-dependent beam profile. This effect will introduce a frequency dependence to the focal spot formed by the LDPE lens in the absence of other effects. A method has recently been presented to determine the spot size of a truncated Gaussian beam focused by a lens. ${ }^{9}$ Application of this treatment using the measured beam diameters above yields a frequency dependent $1 / e$ focal spot diameters of $4.53,2.67$, and $2.32 \mathrm{~mm}$ at $0.5,1$, and $2 \mathrm{THz}$, respectively. This treatment is depicted as solid lines in Fig. 3 . The disagreement between theory and experiment is considered primarily due to the more complex structure and sharper edges of our beam versus that of a Gaussian. In this regard the arrows on the beam profiles for 1 and $2 \mathrm{THz}$ mark the positions of the first null of the diffraction pattern of a uniformly illuminated circular aperture with diameters of 8.5 and $4.25 \mathrm{~mm}$, respectively. The diameters of the nulls were obtained from the relationship $d_{c}=2.44 f \lambda / D_{e}$, yielding the indicated results of $d_{c}=4.46 \mathrm{~mm}$. The above-mentioned treatment demonstrates that the focusing action of the integrated 2D LDPE lens behaves similarly to a standard 3D element in free space. Furthermore, the focusing action of this lens does not appear to introduce detectable higher-order to AIP license or copyright, see http://apl.aip.org/apl/copyright.jsp 


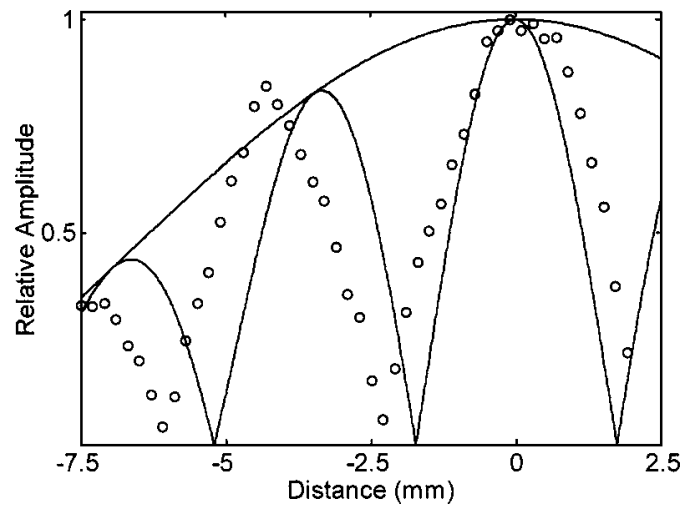

FIG. 4. Measured amplitude diffraction pattern at the output edge from the double slit aperture at $1.5 \mathrm{THz}$ (open circles), calculated double slit diffraction pattern (solid line), and calculated single slit pattern (upper line).

modes within the guide or significantly degrade the performance of the TEM-mode guiding structure.

To further demonstrate the capability of 2D quasioptics, a diffractive element was also demonstrated. The diffractive element is a double slit cut in $12-\mu \mathrm{m}$-thick aluminum foil and attached to the output face of the Si cylindrical lens in close proximity to the 2D LDPE lens. The lens focuses the Fraunhofer diffraction pattern of the double slit aperture onto the output edge. The $5.5-\mathrm{mm}$-long slits are $1.0 \mathrm{~mm}$ in width and are spaced by $3.0 \mathrm{~mm}$ center-to-center. The input and output lenses, as well as the double slit aperture, are held stationary while the remainder of the test structure is scanned along the $x$ axis. The measured spectral amplitude at $1.5 \mathrm{THz}(\lambda=200 \mu \mathrm{m})$, obtained from the corresponding signal pulses, is depicted as the hollow circles in Fig. 4.

The Fraunhofer diffraction pattern of a double slit illuminated with a uniform plane-wave is given by: $E_{\text {diff }}(x)$ $=E_{0}(1 / \alpha) \sin (\alpha) \cos (\delta)$, where $\alpha=(\pi a / \lambda) \sin (\Phi)$ and $\delta$ $=(\pi h / \lambda) \sin (\Phi) . E_{0}$ is the maximum value of the electric field, and is taken as unity, $a$ is the slit width $(1.0 \mathrm{~mm}), h$ is the center-to-center spacing of the slits $(3.0 \mathrm{~mm}), \Phi$ is the angle between a ray extending from the midpoint between slits to an observation point, and the optical axis. The angle $\Phi$ may be obtained from $\Phi=\arctan (x / f)$. For our case the calculated diffraction pattern is shown as the solid line in Fig. 4. The upper line indicates the main peak of the diffraction pattern of a single $1 \mathrm{~mm}$ slit given by $\sin (\alpha) / \alpha$. As shown in the figure, the standard uniform plane-wave assumption adequately predicts the diffraction pattern. Furthermore, the experimental spatial resolution of at least $0.4 \mathrm{~mm}$ is demonstrated by the sharpness of the nulls in the measured data.
The agreement between the predicted diffraction pattern and the measurements can be significantly improved by treating the index of refraction of the LDPE lens as an adjustable parameter. If we reduce the index to $n=1.47$, we obtain precise agreement with experiment for the separation between the nulls in the diffraction pattern of Fig. 4. This treatment likewise yields an improved fit to the consequently broadened 2D foci in Fig. 3. The value of $n=1.47$ is not unreasonable compared to the published bulk value of 1.51 used for the calculations in Figs. 3 and 4. ${ }^{7}$ Furthermore, inconsistencies in the thickness of the LDPE film and the flatness of the copper plates may have resulted in the incorporation of air gaps within the structure serving to reduce the effective index.

The above-described analyses and experiments demonstrate that 2D quasioptical elements placed in a parallel-plate guiding structure work in analogy with their 3D counterparts. The 2D transmissive and diffractive quasioptical elements demonstrated in this work add to the demonstrations in previous work to further illustrate the potential of $\mathrm{THz}$ bandwidth TEM-mode 2D quasioptical structures. The 2D parallel plate structure has been shown to be equivalent to a $2 \mathrm{D}$ optical table for a variety of purposes. For example, these components may be combined to form a rigid structure working as a conventional parallel plate waveguide along one axis, but as a quasioptically coupled guide along the second axis transverse to the direction of propagation. Therefore, these structures show great promise for ultrahighbandwidth interconnect structures at the board and perhaps chip scale. In addition, these 2D quasioptical arrangements may also be used for compact, rugged, guided wave, $\mathrm{THz}$ time-domain spectroscopy applications.

This work was partially supported by the National Science Foundation, the U.S. Army Research Office, and the Semiconductor Research Corporation, Center for Advanced Interconnect Systems Technologies.

\footnotetext{
${ }^{1}$ R. Mendis and D. Grischkowsky, IEEE Microw. Wirel. Compon. Lett. 11, 444 (2001).

${ }^{2}$ R. Mendis and G. Grischkowsky, Opt. Lett. 26, 846 (2001).

${ }^{3}$ S. Coleman and D. Grischkowsky, Appl. Phys. Lett. 83, 3656 (2003).

${ }^{4}$ S. Coleman and D. Grischkowsky, Appl. Phys. Lett. 84, 654 (2004).

${ }^{5}$ S. Zeisberg, A. Schuenemann, G. P. Monahan, P. L. Heron, M. B. Steer, J. W. Mink, and F. K. Schwering, IEEE Microw. Guid. Wave Lett. 3, 253 (1993).

${ }^{6}$ D. Grischkowsky, IEEE J. Sel. Top. Quantum Electron. 6, 1122 (2000).

${ }^{7}$ J. Birch, Infrared Phys. 30, 195 (1990).

${ }^{8}$ M. T. Reiten, S. A. Harmon, and R. A. Cheville, J. Opt. Soc. Am. B 20, 2215 (2003).

${ }^{9}$ H. Urey, Appl. Opt. 43, 620 (2004).
} 